\title{
Comparison of the therapeutic effects of adipose-derived and bone marrow mesenchymal stem cells on erectile dysfunction in diabetic rats
}

\author{
SANSAN CHEN ${ }^{1,2^{*}}$, JIANBIN ZHU $^{3 *}$, MINGZHU WANG $^{4 *}$, YANTING HUANG $^{5}$, ZHUOLIN QIU $^{4}$, \\ JINGJING LI ${ }^{3}$, XINGLU CHEN ${ }^{5}$, HUIYING CHEN ${ }^{2}$, MINGYU XU $^{2}$, JUN LIU $^{6}$, MIAOQIN SHE $^{7}$, \\ HONGWEI LI ${ }^{2}$, XIAORONG YANG ${ }^{5}$, YI WANG ${ }^{8}$ and XIANGSHENG CAI ${ }^{2,5}$
}

\begin{abstract}
${ }^{1}$ Department of Urology, The First Affiliated Hospital of Guangdong Pharmaceutical University, Guangzhou, Guangdong 510080; ${ }^{2}$ Institute of Biotherapy, Southern Medical University, Guangzhou, Guangdong 510515; ${ }^{3}$ Technology Center, Guangdong Vitalife Bio-Tech Co., Ltd., Foshan, Guangdong 528200; ${ }^{4}$ Reproductive Center of Obstetrics and Gynecology, Southern Medical University, Guangzhou, Guangdong 510515; ${ }^{5}$ Clinical Laboratory,

The First Affiliated Hospital of Guangdong Pharmaceutical University, Guangzhou, Guangdong 510080;

${ }^{6}$ Dermatology Hospital of Southern Medical University, Guangzhou, Guangdong 510091; ${ }^{7}$ Guangzhou Institute of Biomedicine and Health, Chinese Academy of Sciences, Guangzhou, Guangdong 510660; ${ }^{8}$ Central Laboratory, The First Affiliated Hospital of Guangdong Pharmaceutical University, Guangzhou, Guangdong 510080, P.R. China
\end{abstract}

Received November 24, 2018; Accepted June 13, 2019

DOI: $10.3892 / \mathrm{ijmm} .2019 .4254$

\begin{abstract}
The aim of the present study was to compare the effects of adipose-derived mesenchymal stem cell (ADSC) and bone marrow mesenchymal stem cell (BMSC) transplantation into the corpora cavernosa of diabetic rats with erectile function. ADSCs and BMSCs were isolated and identified by flow cytometry. Rats with streptozocin-induced diabetes were screened using apomorphine to obtain a rat model of diabetic erectile dysfunction, followed by transplantation of ADSCs and BMSCs into the corpora cavernosa. Two weeks later, the rats were again injected with apomorphine, the intracavernous pressure (ICP) and mean arterial pressure (MAP) of the penile tissue were measured, and the corpus cavernosum tissues were harvested. Angiogenic endothelial nitric oxide synthase (eNOS) expression was detected by western blotting and immunofluorescence analysis. The blood vessels in the corpus cavernosum were observed following hematoxylin and eosin (H\&E) staining, and the expression of collagen was detected by
\end{abstract}

Correspondence to: Dr Xiangsheng Cai or Dr Xiaorong Yang, Clinical Laboratory, The First Affiliated Hospital of Guangdong Pharmaceutical University, 39 Nonglin Xia Road, Guangzhou, Guangdong 510080, P.R. China

E-mail: xiangshengcai@yeah.net

E-mail: 12242818@qq.com

*Contributed equally

Key words: adipose-derived stem cells, bone marrow stem cells, erectile dysfunction, diabetes
Sirius Red staining. The cellular ultrastructure was examined by transmission electron microscopy. Intracavernous injection of ADSCs significantly increased ICP and ICP/MAP. Western blotting and immunofluorescence results revealed that ADSC treatment improved the expression of eNOS in the penile tissue of diabetic rats. The H\&E staining results demonstrated that ADSC treatment promoted revascularization of the corpus cavernosum, and the results of Sirius Red staining revealed that ADSC treatment reduced penile collagen in diabetic rats. Transmission electron microscopy examination revealed that the ultrastructure of the tissues in the ADSC-treated group was more complete compared with that in the untreated diabetic model group. In conclusion, ADSCs were found to be more effective compared with BMSCs in treating diabetes-related erectile dysfunction.

\section{Introduction}

Male erectile dysfunction (ED) poses a serious threat to the quality of life, particularly in older men, causing extreme morbidity among elderly and diabetic patients. ED caused by diabetes is a refractory symptom. The incidence of diabetes mellitus-induced ED (DMED) is $19.0-86.3 \%$, which is three times the rate of ED in non-diabetic patients $(1,2)$. The mechanism of DMED is not entirely clear, but it is associated with problems in blood vessels, nerves, neurotransmitters, and the endocrine system, among others (3-5). Vascular lesions caused by diabetes may induce atheromatous plaque formation in the cavernous arteries of the penis, resulting in reduced blood flow and structural or functional destruction of arterioles and sinusoidal endothelial cells in the cavernous body, further affecting erectile function. Phosphodiesterase 5 inhibitors, which are often used to treat ED in the general population, 
have almost no therapeutic effect on DMED (6). There is thus a clear need for a new approach to DMED treatment.

Adipose-derived mesenchymal stem cells (ADSCs) are multipotent adult stem cells derived from adipose tissue (7). They have the advantages of easy extraction, simple culture and rapid proliferation, and they have become an important type of seed cell for use in regenerative medicine (8). Some scientists have used ADSCs to treat diabetic varicose veins by reprogramming them into endothelial cells that promote capillary formation. In addition, ADSCs have anti-inflammatory properties and can repair nerve damage $(9,10)$. ADSC transplantation therapy is widely used to treat diseases such as myocardial ischemia. Studies have shown that ADSCs can reduce myocardial infarction area, promote angiogenesis and reverse ischemic injury disruption of cardiac remodeling $(11,12)$. ADSCs have also been used to treat a variety of animal models of erectile dysfunction, including DMED models, penile cavernous nerve injury models, penile induration models, and penile cavernous radiation injury models (13-16).

Bone marrow mesenchymal stem cells (BMSCs) are a type of pluripotent stem cells originating from the mesoderm. They are able to differentiate into bone, cartilage and fat (17). Autologous bone marrow transplantation can avoid the complications caused by transplant rejection, but research on and application of this technique are costly (18). Bivalacqua et al (19) cultured BMSCs in vitro, transfected them with angiogenic endothelial nitric oxide synthase (eNOS), and injected these BMSCs into the corpora cavernosa of aged male rats. Histological analysis revealed that BMSCs survived at least 21 days in the corporal tissue and did not cause an obvious inflammatory response. Studies have demonstrated that BMSCs can improve erectile function in older rats $(20,21)$. However, BMSCs are difficult to obtain, and the limited number of available BMSCs cannot meet the clinical demand.

Selecting the optimal type of stem cells to treat DMED is crucial. To date, there is little research comparing the therapeutic effects of ADSCs and BMSCs on diabetic erectile dysfunction. In the present study, a rat model of diabetes was established, the rats were treated with ADSCs and BMSCs, and the therapeutic effects of both types of stem cells in the treatment of DMED were evaluated.

\section{Materials and methods}

Reagents. CD34, CD45, CD73, CD90 and CD105 antibodies were purchased from BD Biosciences. Streptozotocin, apomorphine (APO) and vitamin $\mathrm{C}$ were purchased from Sigma-Aldrich; Merck KGaA. Anti-eNOS and $\beta$-actin antibodies were purchased from Cell Signaling Technology, Inc. Modified Lillie-Mayer hematoxylin staining solution, ethanol eosin staining solution and Sirius Red staining solution were purchased from Reagan.

Male Sprague-Dawley (SD) rats were provided by Guangdong Pharmaceutical University. All animal experiments were approved by the Animal Ethics Committee of Guangdong Pharmaceutical University.

Isolation and culture of rat ADSCs and BMSCs. Male 6-week-old SD rats were anesthetized, sacrificed by cervical dislocation and soaked in iodophor for $2 \mathrm{~min}$, followed by $75 \%$ alcohol for $5 \mathrm{~min}$. Following removal of the epididymal fat, type 1 collagenase was used to digest and separate ADSCs.

To isolate BMSCs, the femur, tibia, and fibula were separated, and the medullary cavity was rinsed with $0.9 \%$ saline solution until the rinsing liquid was clear and the bones were whitish. The rinsing liquid was centrifuged, the supernatant was discarded, and the serum-containing medium was added to resuspend cells.

Identification of ADSCs and BMSCs by flow cytometry. ADSCs and BMSCs were washed once with $0.01 \mathrm{~mol} / 1$ phosphate-buffered saline (PBS) and mixed with CD34, CD45, CD73, CD90 and CD105 monoclonal antibodies at a working concentration of 1:100. The resultant solutions were incubated at $4^{\circ} \mathrm{C}$ for $30 \mathrm{~min}$ and washed twice with $0.01 \mathrm{~mol} / 1$ PBS, after which time ADSCs and BMSCs were detected by flow cytometry.

Establishment and stem cell treatments of diabetic rat models. After 1 week of adaptive feeding, 40 male SD rats were randomly divided into two groups (control group, $n=10$; and diabetes group, $\mathrm{n}=30$ ). The rats in the control group (Ctrl) were provided with a normal diet, while the diabetic rats were fed a high-fat, high-sugar diet. One month later, the diabetic rats were intraperitoneally injected with streptozotocin $(40 \mathrm{mg} / \mathrm{kg})(22)$. Blood glucose was monitored starting 3 days after streptozotocin injection. The animals were considered as diabetic when the glucose levels were $>16.7 \mathrm{mM}$ for 3 consecutive days (reference range, 5-8 $\mathrm{mM}$ ).

The 30 diabetic rats were randomly divided into three groups ( $\mathrm{n}=10$ per group): One group was treated with ADSCs (AD group), one with BMSCs (BM group), and one with PBS alone (PBS group).

Injection sera were created by resuspending ADSCs and BMSCs $\left(1 \times 10^{7}\right.$ cells) in $1 \mathrm{ml}$ of PBS. Rats were anesthetized with pentobarbital $(50 \mathrm{mg} / \mathrm{kg}$, i.p.), and injected with the cell suspensions at the mid-penile corpus cavernosum (23). The AD group rats were injected with $100 \mu$ l of ADSC suspension $\left(1 \times 10^{6}\right.$ cells), the BM group rats were injected with $100 \mu \mathrm{l}$ of BMSC suspension, and animals in the PBS group were injected with $100 \mu 1$ of PBS. The control group did not receive any treatment.

APO-induced erection. APO was used to induce penile erections in rats before and at 4 weeks after stem cell transplantation. The rats were placed in a dark room under observation, kept calm, and allowed to adapt to the environment for $10 \mathrm{~min}$. They were then subcutaneously injected with APO $(100 \mu \mathrm{g} / \mathrm{kg})$ in the back of the neck, and the number of erections in $30 \mathrm{~min}$ was recorded, as measured by intracavernous pressure (ICP) and mean arterial pressure (MAP). Healthy 14-week-old rats were used as controls.

Measurement of ICP and MAP. ICP and MAP were measured as previously described (24). The stimulus parameters were $20 \mathrm{~Hz}$, a pulse width of $0.2 \mathrm{~m} \mathrm{sec}, 1.5 \mathrm{~mA}$, and a duration of $50 \mathrm{sec}$. The ratio of maximum ICP $(\mathrm{mmHg})$ to MAP $(\mathrm{mmHg})$ was calculated to normalize variations in systemic blood pressure. 

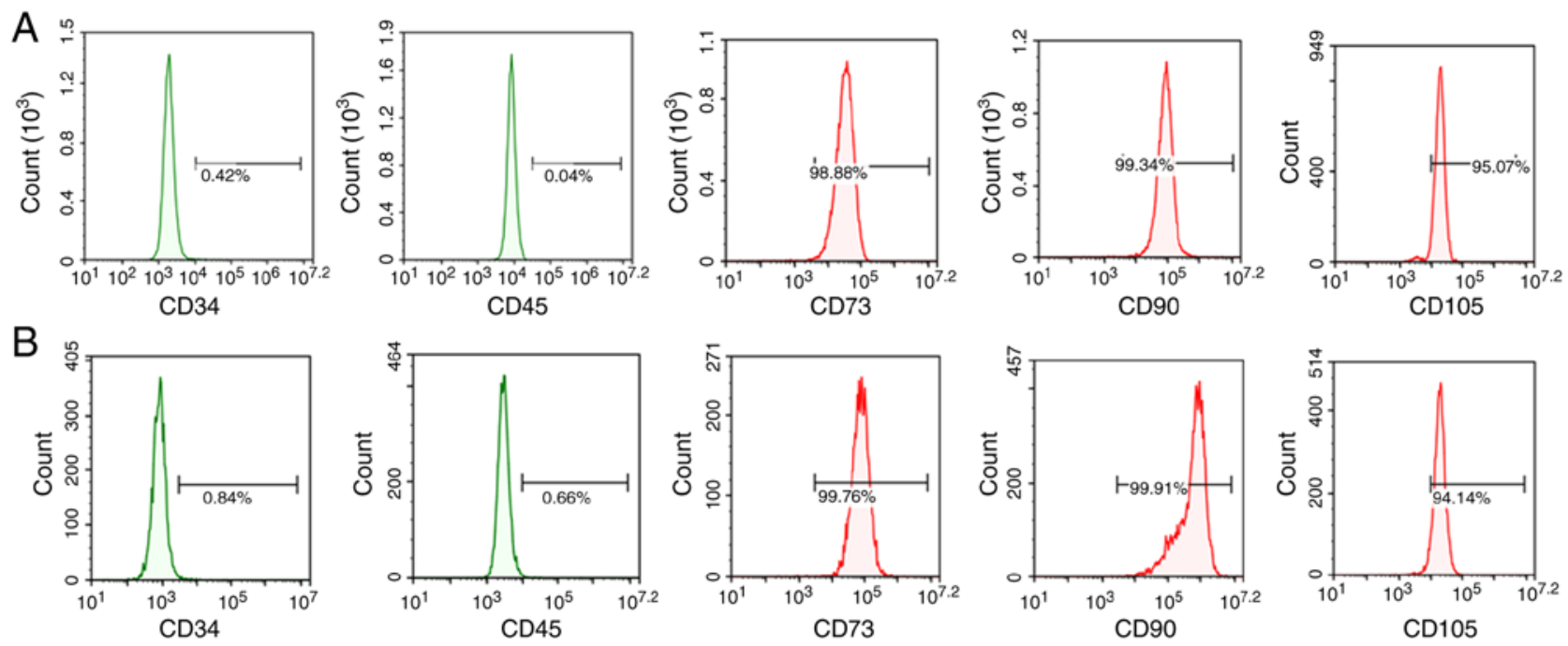

Figure 1. Isolation and characterization of ADSCs and BMSCs. (A) Cells positive for ADSC markers and negative for hematopoietic/endothelial markers. (B) Cells positive for BMSC markers and negative for hematopoietic/endothelial markers. Scale bar=100 $\mu \mathrm{m}$. ADSCs, adipose-derived stem cells; BMSCs, bone marrow-derived stem cells.

Immunofluorescence analysis. After the completion of the APO experiment, five animals from each group were randomly selected, anesthetized and sacrificed by cervical dislocation. Half of the penile tissue from each subject $(10 \mu \mathrm{m})$ was dehydrated in $30 \%$ sucrose and then frozen. After the sections were completely dried, immunofluorescence staining for eNOS was performed, using the penile tissue of normal rats as a control. At least five random fields per section were examined, and the semi-quantitative evaluations were analyzed with Image-Pro Plus software, version 6.0 (Media Cybernetics, Inc.).

Western blot analysis. The remaining half of the penile tissue from each subject was combined with an appropriate amount of pre-cooled RIPA lysis buffer (Fude) and homogenized at $4^{\circ} \mathrm{C}$ to extract proteins from the tissue. The extracted proteins were denatured and used to detect the expression of eNOS by western blotting. The protein expression levels of $\beta$-actin served as the housekeeping control. Images were captured with Tanon-5200 and quantified using Gel-pro 6.0 software.

Hematoxylin and eosin (H\&E) staining and Sirius Red staining. The remaining 5 animals in each group were sacrificed by cervical dislocation, and the middle section of penile tissue was removed. Half of this tissue from each subject was fixed and dehydrated. Paraffin-embedded sections were stained with H\&E or Sirius Red. The numbers of blood vessels and the levels of fibrosis in each group were compared. Images were captured using an Olympus microscope (Olympus Corporation). At least five random fields per section were examined, and semi-quantitative evaluations were performed with Image-Pro Plus software, version 6.0.

Transmission electron microscopy. The remaining half of the penile tissue from each subject was fixed in $2 \%$ glutaraldehyde. The tissue was washed with PBS, followed by $1 \%$ citric acid for $2 \mathrm{~h}$ at room temperature. The tissue was then dehydrated, embedded in paraffin, and cut into ultrathin sections. After double staining tissue samples with uranyl acetate and lead citrate, the morphology of endothelial cells and smooth muscle cells in the cavernous tissue was observed under a transmission electron microscope.

Statistical analysis. All analyses were performed with the GraphPad Prism 4.0 software (GraphPad Software, Inc.). Data are expressed as the mean \pm standard deviation and then analyzed using one-way ANOVA followed by Tukey's multiple comparisons test. P-values $<0.05$ were considered to indicate statistically significant differences.

\section{Results}

Identification of ADSCs and BMSCs by flow cytometry. To determine whether the isolated cells were MSCs, cell surface marker expression was examined. The isolated cells expressed the known MSC markers CD73, CD90 and CD105, but not the hematopoietic and endothelial markers CD34 and CD45 (Fig. 1). Flow cytometry results revealed that the positive rates of CD73, CD90 and CD105 expression in ADSCs were 98.88, 99.34 and $95.07 \%$, respectively, while the positive rates of CD34 and CD45 were 0.42 and $0.04 \%$, respectively. The positive rates of CD73, CD90, and CD105 in BMSCs were 99.76, 99.91 and $94.14 \%$, respectively, and the positive rates of CD34 and CD45 were 0.84 and $0.66 \%$, respectively.

Characteristics of the animals. The mean body weight of the diabetic rats was significantly lower $(\mathrm{P}<0.01)$ compared with that of the normal and ADSC-treated diabetic rats (Table I and Fig. 2A). No significant differences in body weight were observed between the diabetic and the AD or BM groups. The fasting blood glucose levels were significantly higher $(\mathrm{P}<0.01)$ in diabetic rats compared with controls (Table I and Fig. 2B). No significant differences in blood glucose levels were observed between the diabetic group and the AD or BM groups.

Effect of ADSCs and BMSCs on erectile function. The $\mathrm{ICP} / \mathrm{MAP}$ ratios and total ICP values for all groups are 
Table I. Characteristic parameters and functional responses in different treatment groups.

\begin{tabular}{lcccc}
\hline & \multicolumn{3}{c}{ Groups } \\
\cline { 2 - 5 } Parameters & Ctrl & PBS & BM & AD \\
\hline Body weight $(\mathrm{g})$ & $366 \pm 13$ & $280 \pm 10^{\mathrm{a}}$ & $292 \pm 11$ & $304 \pm 9$ \\
Glucose $(\mathrm{mM})$ & $6.5 \pm 0.3$ & $26.4 \pm 0.9^{\mathrm{a}}$ & $24.6 \pm 1.3$ & $23.7 \pm 1.4$ \\
ICP (mm Hg; 7.5 V) & $87 \pm 5$ & $51 \pm 3^{\mathrm{a}}$ & $68 \pm 2$ & $83 \pm 5$ \\
ICP/MAP (7.5 V) & $0.82 \pm 0.02$ & $0.44 \pm 0.03^{\mathrm{a}}$ & $0.62 \pm 0.01$ & $0.71 \pm 0.02$ \\
\hline
\end{tabular}

${ }^{\mathrm{a}} \mathrm{P}<0.01$ vs. control values by analysis of variance and Bonferroni post hoc test. Data are presented as mean \pm standard error of the mean for 10 rats. ICP, intracavernous pressure; MAP, mean arterial pressure; AD, ADSC-treated group; BMSC, bone marrow-derived stem cell; ADSC, adipose-derived stem cell; PBS, phosphate-buffered saline; Ctrl, control.
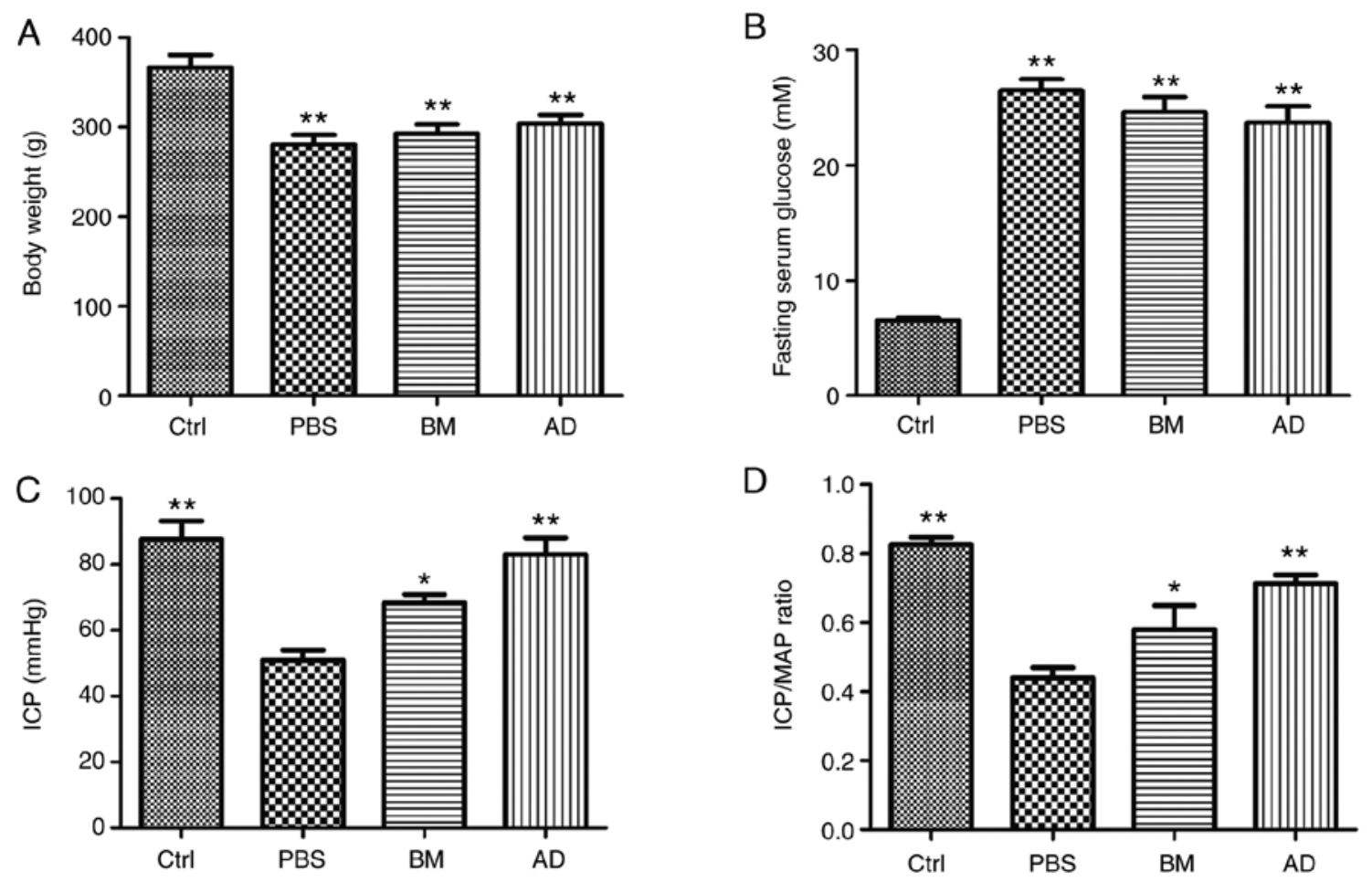

Figure 2. Effects of ADSCs and BMSCs treatments on erectile function in diabetic rats. (A) Body weight in all groups 2 weeks after treatment. (B) Blood glucose levels in all groups 2 weeks after treatment. (C and D) Ratios of total intracavernous pressure (ICP; area under the curve) and maximal ICP to mean arterial pressure (MAP) are recorded. Each bar depicts the mean \pm standard deviation from 5 animals per group. "P $<0.05$ and ${ }^{* *} \mathrm{P}<0.01$ compared with the control (Ctrl) group (non-diabetic rats). The animals in the PBS group were diabetic rats treated with PBS alone. ADSCs, adipose-derived stem cells; BMSCs, bone marrow-derived stem cells; PBS, phosphate-buffered saline; BM, BMSC-treated group; AD, ADSC-treated group.

presented in Fig. 2C and Table I. The ICP/MAP ratios of diabetic rats were lower compared with those of normal rats $(\mathrm{P}<0.01)$, a phenomenon that was reversed through treatment with ADSCs $(\mathrm{P}<0.01)$ or BMSCs $(\mathrm{P}<0.05)$. The ICP/MAP ratios of ADSC-treated diabetic rats were higher compared with those of BMSC-treated diabetic rats. The total ICP values produced by stimulation of the cavernous nerve were lower in diabetic rats compared with normal rats $(\mathrm{P}<0.01)$, but returned to normal levels following treatment with ADSCs $(\mathrm{P}<0.01)$ or BMSCs $(\mathrm{P}<0.05)$. The total ICP values of ADSC-treated diabetic rats were higher compared with those of BMSC-treated diabetic rats.
ADSCs improve the expression of eNOS in the penile tissue of diabetic rats. The expression of eNOS in the penile tissues of the four groups was determined by western blot analysis. As shown in Fig. 3, the expression of eNOS was significantly lower in the diabetic group compared with that in the control group $(\mathrm{P}<0.05)$. The expression of eNOS was higher in the $\mathrm{AD}$ and $\mathrm{BM}$ groups compared with that in the diabetic group $(\mathrm{P}<0.05)$, and slightly higher in the $\mathrm{AD}$ group compared with that in the $\mathrm{BM}$ group.

eNOS expression in the penile tissue was also compared across groups by immunofluorescence analysis, which yielded results similar to those of western blotting: The expression of eNOS was significantly lower in the diabetic group compared with that in the control group $(\mathrm{P}<0.05)$, higher in the $\mathrm{AD}$ 
A

$$
\frac{\mathrm{PBS}}{1 \quad 2} \quad \frac{\mathrm{BM}}{1 \quad 2} \quad \frac{\mathrm{AD}}{1 \quad 2} \quad \frac{\mathrm{Ctrl}}{1 \quad 2}
$$

e-NOS

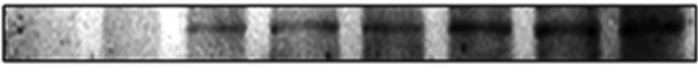

$\beta$-actin
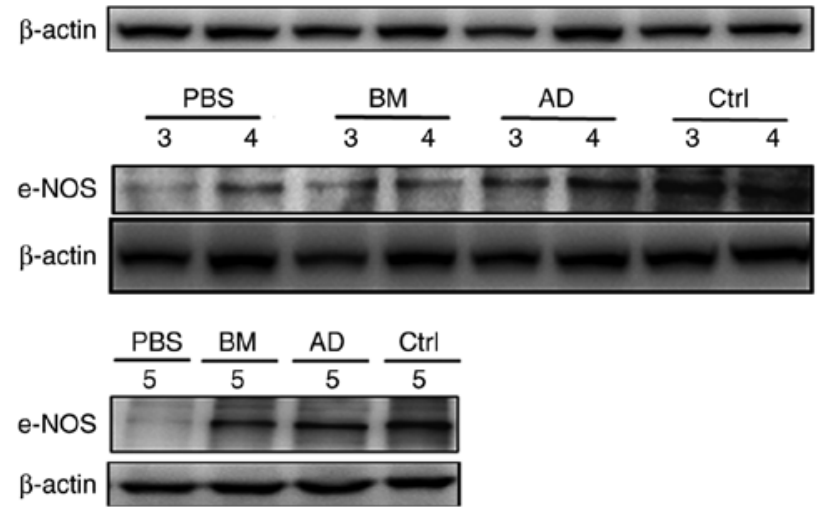

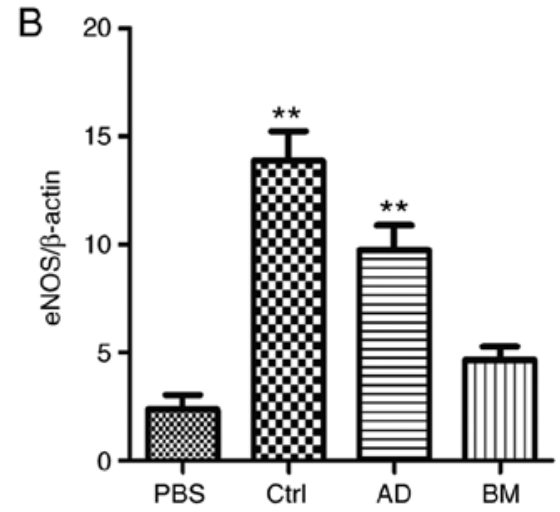

Figure 3. ADSC treatment had obvious restorative effects on eNOS expression. (A) Western blot analysis of eNOS expression. 1 to 5, five samples. (B) Quantitative analysis of eNOS expression in cavernous tissue. Each bar depicts the mean \pm standard deviation values from 5 animals per group. ${ }^{* *} \mathrm{P}<0.01$ compared with the control (Ctrl) group. ADSC, adipose-derived stem cell; BMSC, bone marrow-derived stem cell; PBS, phosphate-buffered saline; BM, BMSC-treated group; AD, ADSC-treated group; eNOS, endothelial nitric oxide synthase.
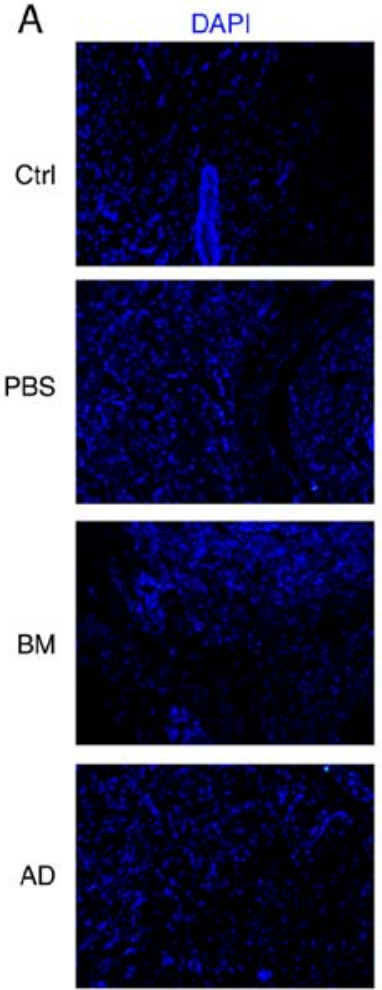
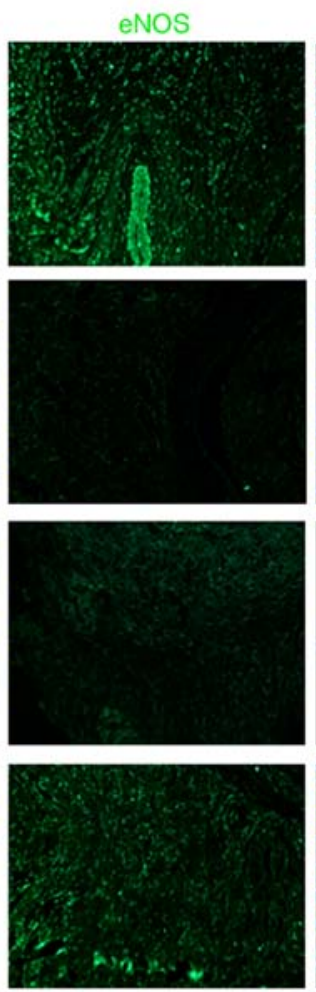
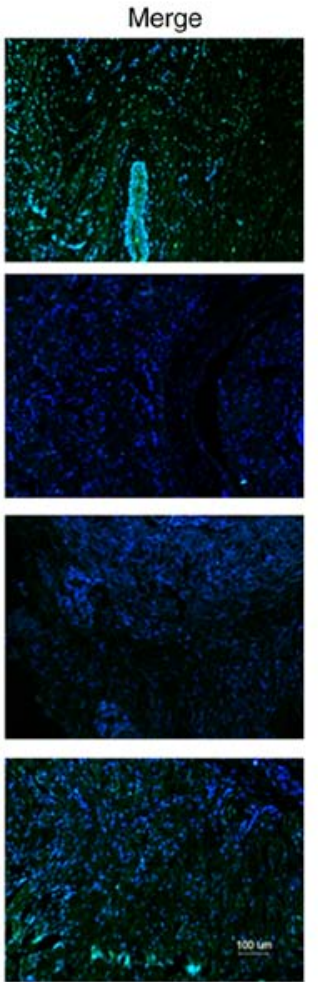

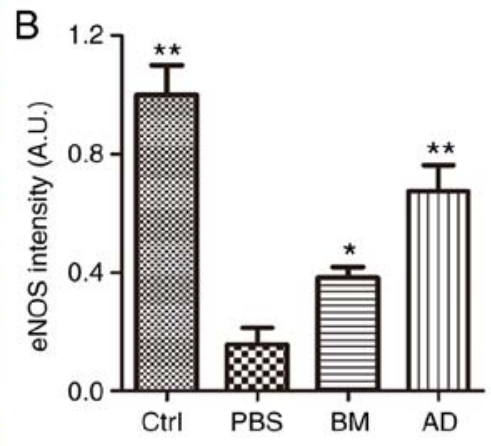

Figure 4. Immunofluorescence staining to detect eNOS expression. (A) The expression of eNOS decreased in the PBS group, while ADSC treatment and BMSC treatment exerted obvious and significant restorative effects on eNOS expression. Scale bar $=100 \mu \mathrm{m}$. (B) Quantitative analysis of eNOS expression in cavernous tissue was performed using an image analyzer. Each bar depicts the mean \pm standard deviation values from 5 animals per group. ${ }^{*} \mathrm{P}<0.05$ and ${ }^{* *} \mathrm{P}<0.01$ compared with the control (Ctrl) group. ADSC, adipose-derived stem cell; BMSC, bone marrow-derived stem cell; PBS, phosphate-buffered saline; $\mathrm{BM}$, BMSC-treated group; AD, ADSC-treated group; eNOS, endothelial nitric oxide synthase.

and $\mathrm{BM}$ groups compared with that in diabetic rats $(\mathrm{P}<0.05)$, and slightly higher in ADSC-treated rats compared with BMSC-treated rats (Fig. 4).

ADSC treatment promotes revascularization in the corpus cavernosum of diabetic rats. H\&E staining was used to observe the number of blood vessels in the corpus cavernosum. As shown in Fig. 5, diabetic rats had significantly fewer blood vessels compared with healthy controls $(\mathrm{P}<0.01)$. This effect was reversed by treatment with ADSCs $(\mathrm{P}<0.01)$ or BMSCs $(\mathrm{P}<0.05)$. The number of blood vessels in ADSC-treated diabetic rats was higher compared with that in BMSC-treated diabetic rats. 
A

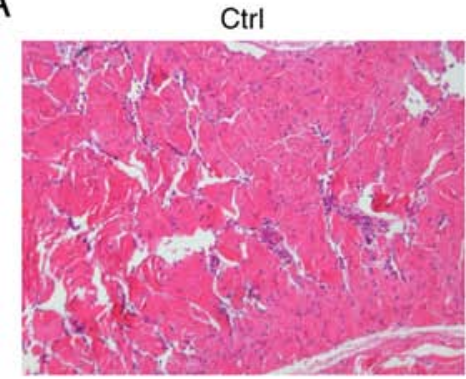

$A D$

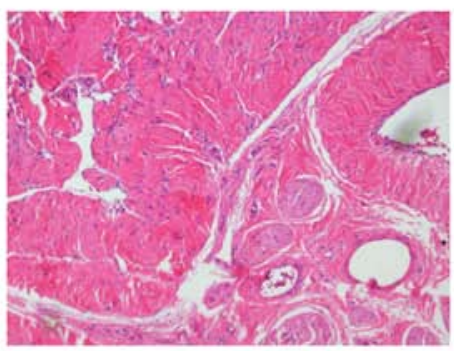

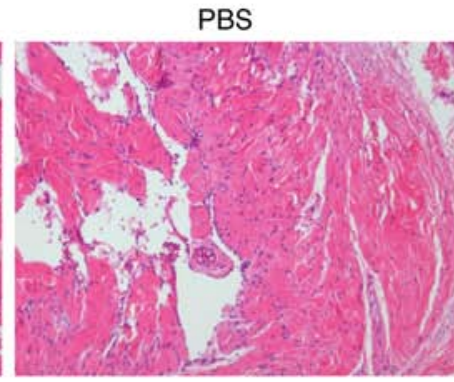

BM

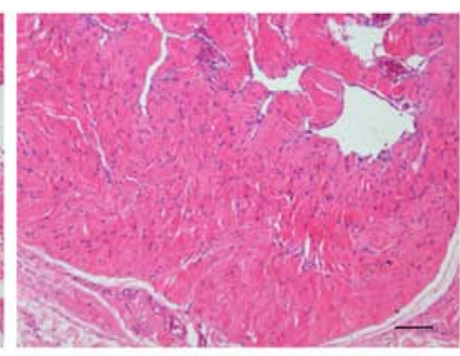

B

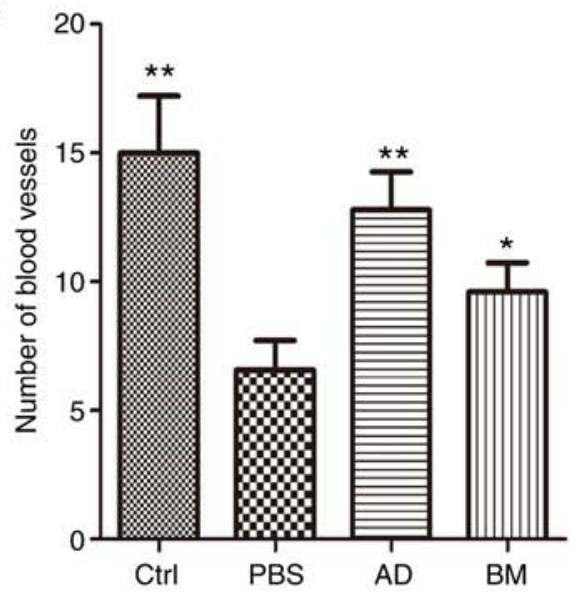

Figure 5. ADSC treatment increased the number of blood vessels in the corpus cavernosum. (A) Hematoxylin and eosin staining of cavernous tissue from diabetic rats and age-matched controls 2 weeks after receiving a single intracavernous injection of PBS, ADSCs, or BMSCs. Scale bar=100 $\mu \mathrm{m}$. (B) Quantitative analysis of the number of blood vessels in cavernous tissue. Each bar depicts the mean \pm standard deviation values from 5 animals per group. ${ }^{*} \mathrm{P}<0.05$ and ${ }^{* *} \mathrm{P}<0.01$ compared with the control (Ctrl) group. ADSC, adipose-derived stem cell; BMSC, bone marrow-derived stem cell; PBS, phosphate-buffered saline; BM, BMSC-treated group; AD, ADSC-treated group.

ADSC treatment reduces collagen content in the corpus cavernosum of diabetic rats. Collagen fiber content in the penile tissue of diabetic rats was investigated using Sirius Red staining. As shown in Fig. 6, the penile tissue of diabetic rats exhibited a significantly increased collagen content, which was reversed by treatment with ADSCs $(\mathrm{P}<0.01)$ or BMSCs $(\mathrm{P}<0.05)$. The collagen fiber content in ADSC-treated diabetic rats was lower compared with that in BMSC-treated diabetic rats.

ADSC treatment ameliorates cell impairment in penile tissue, as observed on transmission electron microscopy. The ultrastructural characteristics of penile tissue cells were observed by transmission electron microscopy. In the corpora cavernosa of diabetic rats, endothelial cells and smooth muscle cells were damaged (Fig. 7), with injuries to the plasma membrane, concentrated cytoplasm and condensed nuclei. Stem cell treatment ameliorated cellular impairments induced by diabetes; this effect was more obvious in the ADSC-treated group compared with that in the BMSC-treated group.

\section{Discussion}

There have been major advances in ED research over the past decade (25), but DMED remains difficult to treat. Stem cell therapy has been proposed as a viable treatment strategy, and some clinical and preclinical trials have been conducted in a few related areas $(26,27)$. A small number of studies have examined the use of ADSCs and BMSCs in the treatment of DMED (28-30) but, to the best of our knowledge, there is currently no report comparing the effects of ADSCs and BMSCs on DMED. Therefore, in the present study, a diabetic rat model was established by streptozocin induction, and ADSCs and BMSCs were transplanted into the penile tissue. The effects of ADSC and BMSC treatment were then compared, and the mechanism of action of each type of stem cell was investigated.

ADSCs and BMSCs were successfully isolated, cultured in vitro, and their identity was confirmed by flow cytometry. Diabetic rats were treated with ADSCs and BMSCs, and their erectile function following treatment was determined by their ICP/MAP ratio. The results demonstrated that ADSC treatment restored the erectile function of diabetic rats.

Several studies have reported that ED is induced by inadequate relaxation of the corpus cavernosum with defects in NO production (31). NO is formed from the conversion of L-arginine by NOS, which exists in three isoforms: Endothelial (eNOS), neuronal (nNOS), and inducible (iNOS). eNOS expression has been identified both in the cavernosal endothelium and in smooth muscle cells (32). Endothelium-generated NO appears to be crucial for maintaining an erection (33). Therefore, in the present study, the expression of eNOS in the penile tissues was examined. According to both western blot and immunofluorescence analyses, ADSC treatment increased the expression of eNOS in diabetic rats, and eNOS expression was slightly higher in the ADSC-treated group compared with that in the BMSC group. Accordingly, H\&E staining revealed that ADSC treatment increased the number of blood vessels in the penile tissue of diabetic rats. These findings suggest that ADSC treatment was more effective compared with BMSC treatment in promoting revascularization of the corpus cavernosum in diabetic rats.

In addition, the ultrastructural characteristics of endothelial and smooth muscle cells in each group were observed under a transmission electron microscope. Cellular morphological characteristics suggested that ADSCs were more effective compared with BMSCs in ameliorating diabetes-induced 
A

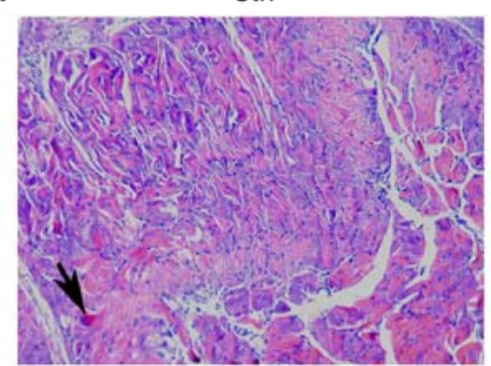

$A D$

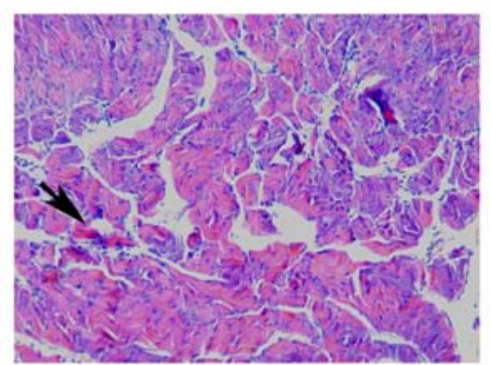

PBS

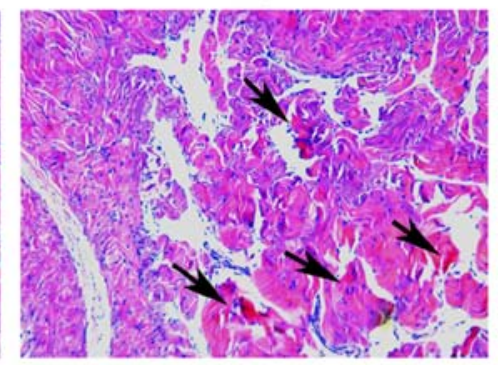

$\mathrm{BM}$

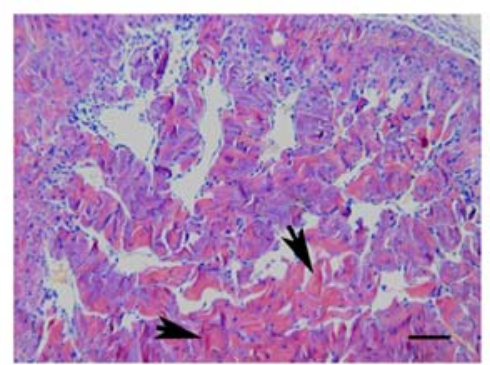

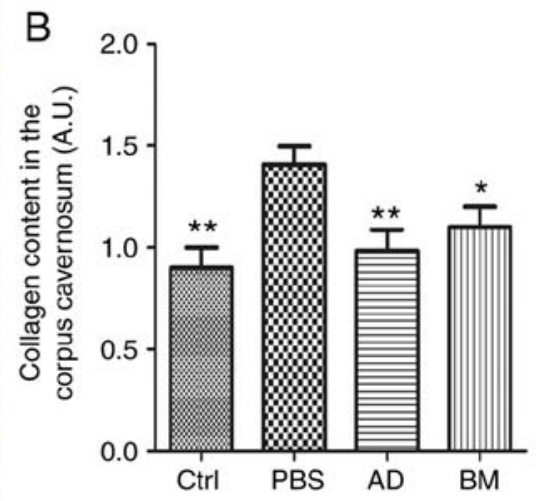

Figure 6. ADSC treatment reduced collagen content in cavernous tissue. (A) Sirius Red staining of cavernous tissue from diabetic rats and age-matched controls 2 weeks after receiving a single intracavernous injection of PBS, ADSCs, or BMSCs. Scale bar=100 $\mu \mathrm{m}$. (B) Quantitative analysis of collagen content in cavernous tissue was performed using an image analyzer. Each bar depicts the mean \pm standard deviation values from 5 animals per group. "P<0.05 and ${ }^{* *} \mathrm{P}<0.01$ compared with the control (Ctrl) group. ADSC, adipose-derived stem cell; BMSC, bone marrow-derived stem cell; PBS, phosphate-buffered saline; $\mathrm{BM}$, BMSC-treated group; AD, ADSC-treated group.
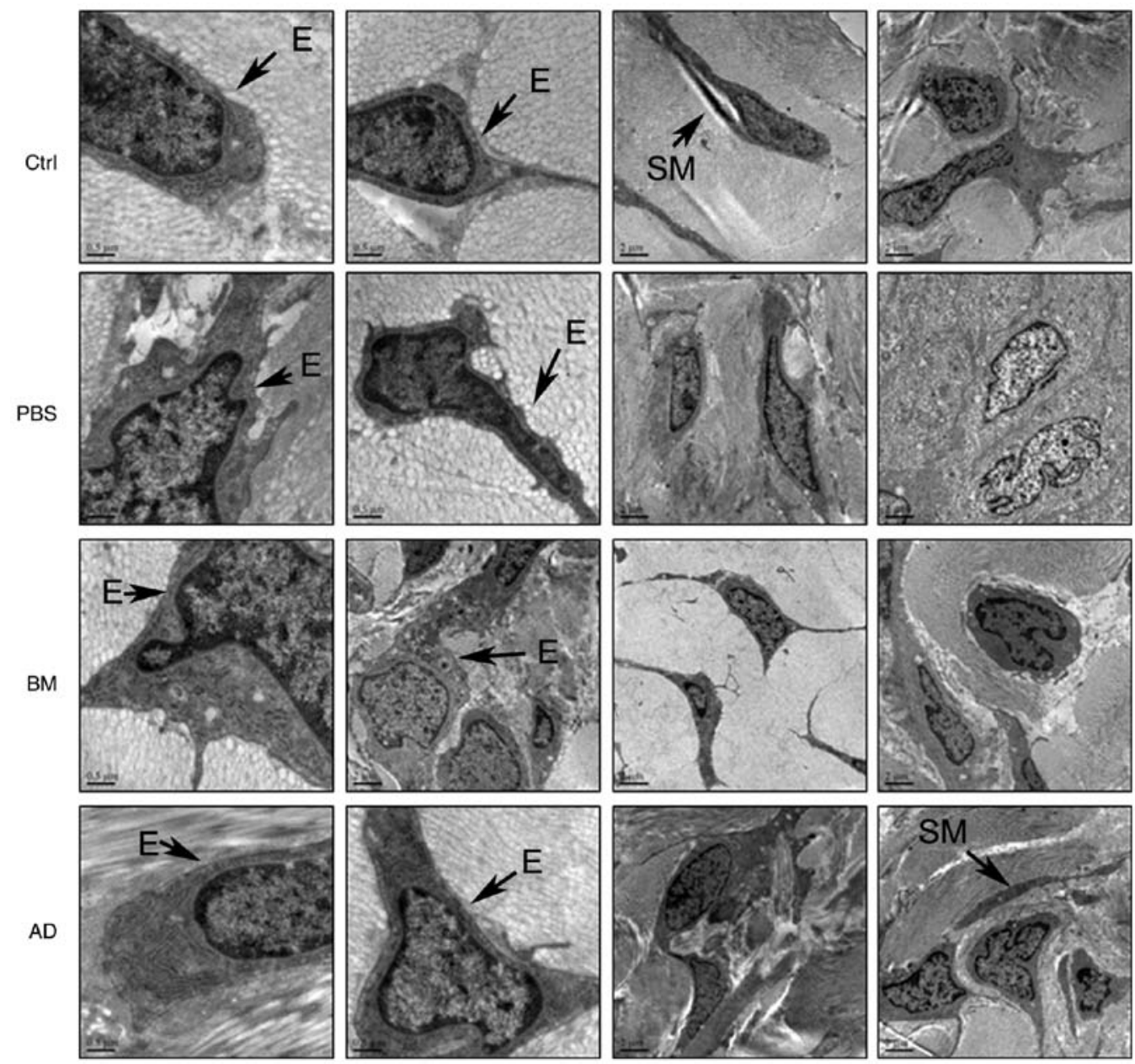

Figure 7. Ultrastructural analysis of endothelial cells and smooth muscle cells in rat corpora cavernosa by transmission electron microscopy. SM, smooth muscle; E, endothelial cells; BM, BMSC-treated group; AD, ADSC-treated group; BMSC, bone marrow-derived stem cell; ADSC, adipose-derived stem cell; PBS, phosphate-buffered saline; Ctrl, control. 
impairment of endothelial and smooth muscle cells in penile tissue.

A mounting number of studies have demonstrated that ADSC treatment can promote revascularization, which suggests that ADSCs are a promising approach to the treatment of diseases such as critical limb ischemia (11), ischemic cardiomyopathy (34) and myocardial infarction (35). ADSCs are adult stem cells isolated from adipose tissue that are capable of self-renewal and multi-directional differentiation (36). They have low immunogenicity and can be easily collected with minimal risk. In the field of regenerative medicine, ADSCs are widely used in tissue repair and regeneration. All these characteristics make ADSCs the most suitable type of stem cell for use in the treatment of ED (37). ADSC transplantation has achieved encouraging results in multiple ED models, including DMED $(38,39)$. There is increasing evidence that the therapeutic effects of ADSCs on ED may be mediated through paracrine indirect effects of growth factors or cytokines, rather than by direct differentiation into specific cell types (40), a subject which requires further study.

Several studies have indicated that BMSC treatment may also promote revascularization $(41,42)$. However, in the present study, ADSCs promoted angiogenesis more effectively compared with BMSCs, which is consistent with the earlier findings of $\mathrm{Wu}$ et al (43). To the best of our knowledge, this is the first study to directly compare the therapeutic effects of ADSCs and BMSCs on ED in diabetic rats.

However, this was a preliminary study that mainly focuses on comparing the therapeutic effects of ADSCs and BMSCs on DMED. On this basis, more in-depth mechanism research is still needed, and experiments for protein or non-coding RNA are required as the next step.

In conclusion, treatment with ADSCs significantly restored erectile function in diabetic rats by improving eNOS expression, promoting revascularization, and reversing cellular functional impairment. ADSC treatment was found to be more effective compared with BMSC treatment in restoring erectile function in diabetic rats. These findings may provide a new approach to the treatment of DMED.

\section{Acknowledgements}

Not applicable.

\section{Funding}

The authors would like to thank the Guangdong Province Science and Technology Fund (grant. no. 2017ZC0208) for their financial support.

\section{Availability of materials and data}

All the datasets generated and analyzed in the present study are available from the corresponding author on reasonable request.

\section{Authors' contributions}

$\mathrm{XC}, \mathrm{YW}$ and $\mathrm{XY}$ conceived and designed the study. SC, JZ and $\mathrm{MW}$ conducted the experiments. YH, ZQ, HC, MX, XC,
JJL and MS participated in the completion of the experiments. JL and HL analyzed the data. SC wrote the manuscript. XC, YW and XY revised the manuscript. All the authors have read and approved the final version of this manuscript for publication.

\section{Ethics approval and consent to participate}

All animal experiments were approved by the Animal Ethics Committee of Guangdong Pharmaceutical University.

\section{Patient consent for publication}

Not applicable.

\section{Competing interests}

The authors declare that they have no competing interests.

\section{References}

1. Jangir RN and Jain GC: Diabetes mellitus induced impairment of male reproductive functions: A review. Curr Diabetes Rev 10: 147-157, 2014.

2. Tamás V and Kempler P: Sexual dysfunction in diabetes. Handb Clin Neurol 126: 223-232, 2014.

3. Sun X, Luo LH, Feng L and Li DS: Down-regulation of lncRNA MEG3 promotes endothelial differentiation of bone marrow derived mesenchymal stem cells in repairing erectile dysfunction. Life Sci 208: 246-252, 2018.

4. Hu LL, Zhang KQ, Tian T, Zhang H and Fu Q: Probucol improves erectile function via Activation of $\mathrm{Nrf} 2$ and coordinates the HO-1/ DDAH / PPAR- $\gamma /$ eNOS pathways in streptozotocin-induced diabetic rats. Biochem Biophys Res Commun 507: 9-14, 2018.

5. Wu Y, Yang C, Meng F, Que F, Xiao W, Rao H, Wan Y, Taylor HS and $\mathrm{Lu} \mathrm{L}$ : Nerve growth factor improves the outcome of type 2 diabetes-induced hypotestosteronemia and erectile dysfunction. Reprod Sci 26: 386-393, 2019.

6. Shamloul R and Ghanem H: Erectile dysfunction. Lancet 381: 153-65, 2013.

7. Xu X, Li L, Wang C, Liu Y, Chen C, Yan J, Ding H and Tang SY: The expansion of autologous adipose-derived stem cells in vitro for the functional reconstruction of nasal mucosal tissue. Cell Biosci 5: 54, 2015.

8. Chen S, Wang M, Chen X, Chen S, Liu L, Zhu J, Wang J, Yang X and Cai X: In vitro expression of Cytokeratin 19 in adipose-derived stem cells is induced by epidermal growth factor. Med Sci Monit 24: 4254-4261, 2018.

9. Liu YP, Li SZ, Yuan F, Xia J, Yu X, Liu X and Yu GR: Infrapatellar fat pad may be with tendon repairing ability and closely related with the developing process of patella Baja. Med Hypotheses 77: 620-623, 2011

10. Gadelkarim M, Abushouk AI, Ghanem E, Hamaad AM, Saad AM and Abdel-Daim MM: Adipose-derived stem cells: Effectiveness and advances in delivery in diabetic wound healing. Biomed Pharmacother 107: 625-633, 2018.

11. Liu J, Zhu P, Song P, Xiong W, Chen H, Peng W, Wang S, Li S, $\mathrm{Fu} Z$, Wang Y and Wang H: Pretreatment of adipose derived stem cells with curcumin facilitates myocardial recovery via antiapoptosis and angiogenesis. Stem Cells Int 2015: 638153, 2015.

12. Burchfield JS, Paul AL, Lanka V, Tan W, Kong Y, McCallister C, Rothermel BA, Schneider JW, Gillette TG and Hill JA: Pharmacological priming of adipose-derived stem cells promotes myocardial repair. J Investig Med 64: 50-62, 2016.

13. Fandel TM, Albersen M, Lin G, Qiu X, Ning H, Banie L, Lue TF and Lin CS: Recruitment of intracavernously injected adipose-derived stem cells to the major pelvic ganglion improves erectile function in a rat model of cavernous nerve injury. Eur Urol 61: 201-210, 2012.

14. Qiu X, Villalta J, Ferretti L, Fandel TM, Albersen M, Lin G, Dai Y, Lue TF and Lin CS: Effects of intravenous injection of adipose-derived stem cells in a rat model of radiation therapy-induced erectile dysfunction. J Sex Med 9: 1834-1841, 2012. 
15. Garcia MM, Fandel TM, Lin G, Shindel AW, Banie L, Lin CS and Lue TF: Treatment of erectile dysfunction in the obese type 2 diabetic ZDF rat with adipose tissue-derived stem cells. J Sex Med 7: 89-98, 2010.

16. Gokce A, Abd Elmageed ZY, Lasker GF, Bouljihad M, Kim H, Trost LW, Kadowitz PJ, Abdel-Mageed AB, Sikka SC and Hellstrom WJ: Adipose tissue-derived stem cell therapy for prevention and treatment of erectile dysfunction in a rat model of Peyronie's disease. Andrology 2: 244-251, 2014.

17. Singh A, Lee D, Sopko N, Matsui H, Sabnekar P, Liu X, Elisseeff J, Schoenberg MP, Pienta K and Bivalacqua TJ: Biomanufacturing seamless tubular and hollow collagen scaffolds with unique design features and biomechanical properties Adv Healthc Mater 6, 2017

18. Abdel-Latif A, Bolli R, Tleyjeh IM, Montori VM, Perin EC, Hornung CA, Zuba-Surma EK, Al-Mallah M and Dawn B: Adult bone marrow-derived cells for cardiac repair: A systematic review and meta-analysis. Arch Intern Med 167: 989-997, 2007.

19. Bivalacqua TJ, Deng W, Kendirci M, Usta MF, Robinson C, Taylor BK, Murthy SN, Champion HC, Hellstrom WJ and Kadowitz PJ: Mesenchymal stem cells alone or ex vivo gene modified with endothelial nitric oxide synthase reverse age-associated erectile dysfunction. Am J Physiol Heart Circ Physiol 292: H1278-H1290, 2007.

20. Ryu JK, Kim DH, Song KM, Yi T, Suh JK and Song SU: Intracavernous delivery of clonal mesenchymal stem cells restores erectile function in a mouse model of cavernous nerve injury. J Sex Med 11: 411-423, 2014.

21. Ryu JK, Kim DH, Song KM, Ryu DS, Kim SN, Shin DH, Yi T, Suh JK and Song SU: Intracavernous delivery of clonal mesenchymal stem cells rescues erectile function in the streptozotocin-induced diabetic mouse. Andrology 4: 172-184, 2016.

22. Cai X, Li J, Wang M, She M, Tang Y, Li J, Li H and Hui H: GLP-1 treatment improves diabetic retinopathy by alleviating autophagy through GLP-1R-ERK1/2-HDAC6 signaling pathway. Int J Med Sci 14: 1203-1212, 2017.

23. Liu G, Sun X, Bian J, Wu R, Guan X, Ouyang B, Huang Y, Xiao H, Luo D, Atala A, et al: Correction of diabetic erectile dysfunction with adipose derived stem cells modified with the vascular endothelial growth factor gene in a rodent diabetic model. PLoS One 8: e72790, 2013.

24. Xu Y, Guan R, Lei H, Li H, Wang L, Gao Z, Song W and Xin Z: Therapeutic potential of adipose-derived stem cells-based micro-tissues in a rat model of postprostatectomy erectile dysfunction. J Sex Med 11: 2439-2448, 2014

25. Decaluwé K, Pauwels B, Boydens C and Van de Voorde J: Treatment of erectile dysfunction: New targets and strategies from recent research. Pharmacol Biochem Behav 121: 146-157, 2014.

26. Zhou F, Hui Y, Xin H, Xu YD, Lei HE, Yang BC, Guan RL, Li M, Hou JQ and Xin ZC: Therapeutic effects of adipose-derived stem cells-based microtissues on erectile dysfunction in streptozotocin-induced diabetic rats. Asian J Androl 19: 91-97, 2017.

27. Hou QL, Ge MY, Zhang CD, Tian DD, Wang LK, Tian HZ Wang WH and Zhang WD: Adipose tissue-derived stem cell therapy for erectile dysfunction in rats: A systematic review and meta-analysis. Int Urol Nephrol 49: 1127-1137, 2017.

28. Chen F, Zhang H, Wang Z, Ding W, Zeng Q, Liu W, Huang C, $\mathrm{He} \mathrm{S}$ and Wei A: Adipose-derived stem cell-derived exosomes ameliorate erectile dysfunction in a rat model of type 2 Diabetes. J Sex Med 14: 1084-1094, 2017.

29. Mangır N and Türkeri L: Stem cell therapies in post-prostatectomy erectile dysfunction: A critical review. Can J Urol 24 $8609-8619,2017$.
30. Shan HT, Zhang HB, Chen WT, Chen FZ, Wang T, Luo JT, Yue M, Lin JH and Wei AY: Combination of low-energy shock-wave therapy and bone marrow mesenchymal stem cell transplantation to improve the erectile function of diabetic rats. Asian J Androl 19: 26-33, 2017.

31. Azadzoi KM, Goldstein I, Siroky MB, Traish AM, Krane RJ and Saenz de Tejada I: Mechanisms of ischemia-induced cavernosal smooth muscle relaxation impairment in a rabbit model of vasculogenic erectile dysfunction. J Urol 160: 2216-2222, 1998.

32. Burnett AL, Lowenstein CJ, Bredt DS, Chang TS and Snyder SH: Nitric oxide: A physiologic mediator of penile erection. Science 257: 401-403, 1992.

33. Andersson KE: Erectile physiological and pathophysiological pathways involved in erectile dysfunction. J Urol 170: S6-S13, 2003.

34. Procházka V, Jurčíková J, Laššák O, Vítková K, Pavliska L, Porubová L, Buszman PP, Krauze A, Fernandez C, Jalůvka F, et al: Therapeutic potential of adipose-derived therapeutic factor concentrate for treating critical limb ischemia. Cell Transplant 25: 1623-1633, 2016.

35. Mazo M, Hernández S, Gavira JJ, Abizanda G, Araña M, López-Martínez T, Moreno C, Merino J, Martino-Rodríguez A, Uixeira A, et al: Treatment of reperfused ischemia with adipose-derived stem cells in a preclinical Swine model of myocardial infarction. Cell Transplant 21: 2723-2733, 2012.

36. Salibian AA, Widgerow AD, Abrouk M and Evans GR: Stem cells in plastic surgery: A review of current clinical and translational applications. Arch Plast Surg 40: 666-675, 2013.

37. Li M, Li H, Ruan Y, Wang T and Liu J: Stem cell therapy for diabetic erectile dysfunction in rats: A meta-analysis. PLoS One 11: e0154341, 2016.

38. Wang X, Liu C, Li S, Xu Y, Chen P, Liu Y, Ding Q, Wahafu W, Hong $B$ and Yang $M$ : Hypoxia precondition promotes adipose-derived mesenchymal stem cells based repair of diabetic erectile dysfunction via augmenting angiogenesis and neuroprotection. PLoS One 10: e0118951, 2015.

39. Liu T, Peng Y, Jia C, Fang X, Li J and Zhong W: Hepatocyte growth factor-modified adipose tissue-derived stem cells improve erectile function in streptozotocin-induced diabetic rats. Growth Factors 33: 282-289, 2015.

40. Albersen M, Weyne E and Bivalacqua TJ: Stem cell therapy for erectile dysfunction: Progress and future directions. Sex Med Rev 1: 50-64, 2013.

41. Liu Y, Yang X, Maureira P, Falanga A, Marie V, Gauchotte G, Poussier S, Groubatch F, Marie PY and Tran N: Permanently hypoxic cell culture yields rat bone marrow mesenchymal cells with higher therapeutic potential in the treatment of chronic myocardial infarction. Cell Physiol Biochem 44: 1064-1077, 2017.

42. Ju X, Xue D, Wang T, Ge B, Zhang Y and Li Z: Catalpol promotes the survival and VEGF secretion of bone marrow-derived stem cells and their role in myocardial repair after myocardial infarction in rats. Cardiovasc Toxicol 18: 471-481, 2018.

43. Wu H, Li JZ, Xie BD, Tian H, Fang SH, Jiang SL and Kang K: Lower senescence of adipose-derived stem cells than donor-matched bone marrow stem cells for surgical ventricular restoration. Stem Cells Dev 27: 612-623, 2018.

(i) $(5)$ This work is licensed under a Creative Commons Attribution-NonCommercial-NoDerivatives 4.0 International (CC BY-NC-ND 4.0) License. 